\title{
Local Regularity Results for Minima of Anisotropic Functionals and Solutions of Anisotropic Equations
}

\author{
Gao Hongya, ${ }^{1,2}$ Qiao Jinjing, ${ }^{1}$ Wang Yong, ${ }^{3}$ and Chu Yuming ${ }^{4}$ \\ ${ }^{1}$ College of Mathematics and Computer Science, Hebei University, Baoding 071002, China \\ ${ }^{2}$ Study Center of Mathematics of Hebei Province, Shijiazhuang 050016, China \\ ${ }^{3}$ Department of Mathematics, Chengde Teachers College for Nationalities, Chengde 067000, China \\ ${ }^{4}$ Faculty of Science, Huzhou Teachers College, Huzhou 313000, China
}

Correspondence should be addressed to Gao Hongya, hongya-gao@sohu.com

Received 13 July 2007; Accepted 21 November 2007

Recommended by Alberto Cabada

This paper gives some local regularity results for minima of anisotropic functionals $I(u ; \Omega)=\int_{\Omega} f(x$, $u, D u) d x, u \in W_{\text {loc }}^{1, q_{i}}(\Omega)$ and for solutions of anisotropic equations $-\operatorname{div} \mathcal{A}(x, u, D u)=-\sum_{i=1}^{N}(\partial f /$ $\left.\partial x_{i}\right), u \in W_{\text {loc }}^{1, q_{i}}(\Omega)$ which can be regarded as generalizations of the classical results.

Copyright $(\odot 2008$ Gao Hongya et al. This is an open access article distributed under the Creative Commons Attribution License, which permits unrestricted use, distribution, and reproduction in any medium, provided the original work is properly cited.

\section{Introduction}

Let $\Omega$ be an open bounded subset of $\mathbf{R}^{N}, N \geq 2$. Let $q_{i}>1, i=1, \ldots, N$. Denote

$$
q=\max _{1 \leq i \leq N} q_{i}, \quad p=\min _{1 \leq i \leq N} q_{i}, \quad \bar{q}: \frac{1}{\bar{q}}=\frac{1}{N} \sum_{i=1}^{N} \frac{1}{q_{i}} .
$$

Throughout this paper, we will make use of the anisotropic Sobolev space

$$
W_{\mathrm{loc}}^{1, q_{i}}(\Omega)=\left\{v \in L_{\mathrm{loc}}^{q}(\Omega): \frac{\partial v}{\partial x_{i}} \in L_{\mathrm{loc}}^{q_{i}}(\Omega), \forall i=1, \ldots, N\right\} .
$$

Let $x_{0} \in \Omega$ and $t>0$, we denote by $B_{t}$ the ball of radius $t$ centered at $x_{0}$. For functions $u$ and $k>0$, let $A_{k}=\{x \in \Omega:|u(x)|>k\}, A_{k, t}=A_{k} \cap B_{t}$. Moreover, if $p>1$, then $p^{\prime}$ is always the real number $p /(p-1)$, and if $s<N, s^{*}$ is always the real number satisfying $1 / s^{*}=1 / s-1 / N$.

This paper mainly considers the functions $u$ minimizing the anisotropic functionals

$$
I(u ; \Omega)=\int_{\Omega} f(x, u, D u) d x, \quad u \in W_{\mathrm{loc}}^{1, q_{i}}(\Omega)
$$


and weak solutions of the anisotropic equations

$$
-\operatorname{div} \mathcal{A}(x, u, D u)=-\sum_{i=1}^{N} \frac{\partial f_{i}}{\partial x_{i}}, \quad u \in W_{\mathrm{loc}}^{1, q_{i}}(\Omega) .
$$

We refer to the classical books by Ladyženskaya and Ural'ceva [1], Morrey [2], Gilbarg and Trudinger [3], and Giaquinta [4] for some details of isotropic cases.

For isotropic cases, global $L^{s}$-summability was proved in the 1960s by Stampacchia [5] for solutions of linear elliptic equations. This result was extended by Boccardo and Giachetti to the nonlinear case in [6]. For anisotropic cases, Giachetti and Porzio recently proved in [7] the local $L^{S}$-summability for minima of anisotropic functionals and weak solutions of anisotropic nonlinear elliptic equations. Precisely, the authors considered the minima of functionals whose prototype is (1.3), $f$ is a Carathéodory function satisfying the growth conditions

$$
a \sum_{i=1}^{N}\left|\xi_{i}\right|^{q_{i}} \leq f(x, s, \xi) \leq b \sum_{i=1}^{N}\left|\xi_{i}\right|^{q_{i}}+\varphi_{1}(x),
$$

where the function $\varphi_{1} \in L_{\text {loc }}^{r}(\Omega)$ with $1<r<N / \bar{q}$. The authors also considered the local solutions $u \in W_{\text {loc }}^{1, q_{i}}(\Omega)$ of the anisotropic equations (1.4), where $\mathcal{A}: \Omega \times \mathbf{R} \times \mathbf{R}^{N} \rightarrow \mathbf{R}^{N}$ is a Caratheodory function satisfying the following structural conditions:

$$
\begin{gathered}
\mathcal{A}(x, u, \xi) \cdot \xi \geq m_{0} \sum_{i=1}^{N}\left|\xi_{i}\right|^{q_{i}}, \\
\left|\mathcal{A}_{j}(x, u, \xi)\right| \leq m_{1}\left(h(x)+\sum_{i=1}^{N}\left|\xi_{i}\right|^{q_{i}}\right)^{1-1 / q_{j}}, j=1, \ldots, N,
\end{gathered}
$$

where $m_{l}, l=0,1$ are positive constants, the function $h$ is in $L_{\text {loc }}^{1}(\Omega)$ and the functions $f_{i}$ belong, respectively, to the spaces $L_{\text {loc }}^{\left(q_{i}\right)^{\prime}}(\Omega)$. Under the above conditions, the authors obtained some local regularity results.

The aim of the present paper is to prove the local regularity property for minima of the anisotropic functionals of type (1.3) with the more general growth conditions than (1.5), that is, we assume the integrand $f$ satisfies the following growth conditions:

$$
\sum_{i=1}^{N}\left|\xi_{i}\right|^{q_{i}}-b|u|^{\alpha}-\varphi_{0}(x) \leq f(x, u, \xi) \leq a \sum_{i=1}^{N}\left|\xi_{i}\right|^{q_{i}}+b|u|^{\alpha}+\varphi_{1}(x),
$$

where

$$
\begin{gathered}
\varphi_{0} \in L_{\mathrm{loc}}^{r_{1}}(\Omega), \quad \varphi_{1} \in L_{\mathrm{loc}}^{r_{2}}(\Omega), \quad r_{1}, r_{2}>1, \quad a \geq 1, b \geq 0, \\
p \leq \alpha<p^{*}, \quad q<\bar{q}^{*}, \quad \bar{q}<N, \quad 1<\min \left\{r_{1}, r_{2}\right\}<\frac{N}{\bar{q}} .
\end{gathered}
$$

We also consider weak solutions of the type (1.4) with more general growth conditions than (1.6), that is, we assume the operator $\mathcal{A}$ satisfies the following coercivity and growth conditions:

$$
\begin{aligned}
& \mathcal{A}(x, u, \xi) \cdot \xi \geq b_{0} \sum_{i=1}^{N}\left|\xi_{i}\right|^{q_{i}}-b_{1}|u|^{\alpha_{1}}-\varphi_{2}(x), \\
& |\mathcal{A}(x, u, \xi)| \leq b_{2} \sum_{i=1}^{N}\left|\xi_{i}\right|^{q_{i}-1}+b_{3}|u|^{\alpha_{2}}+k(x),
\end{aligned}
$$


where $b_{0} \geq 1, b_{i}>0, i=1,2,3, q<\bar{q}^{*}, \bar{q}<N, p \leq \alpha_{1}<p^{*}, p-1 \leq \alpha_{2} \leq N(p-1) /(N-p)$, $\varphi_{2} \in L_{\mathrm{loc}}^{r_{0}}(\Omega)$ with $r_{0}>1, k \in L_{\mathrm{loc}}^{r_{N+1}}(\Omega), f_{i} \in L_{\mathrm{loc}}^{r_{i}}(\Omega), i=1, \ldots, N$.

Remark 1.1. Notice that we have confined ourselves to the case $\bar{q}<N$ because when such inequality is violated, every function in $W_{\text {loc }}^{1, q_{i}}(\Omega)$ is trivially in $L_{\text {loc }}^{s}(\Omega)$ (for every fixed $s<\infty$ ) by [7, Lemma 3.2].

Remark 1.2. Since we have assumed in (1.7), (1.9), and (1.10) that the integrand $f$ and the operator $\mathcal{A}$ satisfy some growth conditions depending on $u$, in the proof of the local regularity results, we have to estimate the integral of some power of $|u|$ by means of $|D u|$. To do this, we will make use of the Sobolev inequality that has been used in [8].

\section{Preliminary lemmas}

In order to prove the local $L^{s}$-integrability of the local unbounded minima of the anisotropic functionals and weak solutions of anisotropic equations, we need a useful lemma from [7].

Lemma 2.1. Let $u \in W_{\mathrm{loc}}^{1, q_{i}}(\Omega), \phi_{0} \in L_{\text {loc }}^{r}(\Omega)$, where $q, \bar{q}$, and $r$ satisfy

$$
1<r<\frac{N}{\bar{q}}, \quad q<\bar{q}^{*}, \quad \bar{q}<N
$$

Assume that the following integral estimates hold:

$$
\int_{A_{k, \tau}} \sum_{i=1}^{N}\left|\frac{\partial u}{\partial x_{i}}\right|^{q_{i}} d x \leq c_{0}\left[\int_{A_{k, t}} \phi_{0} d x+(t-\tau)^{-\gamma} \int_{A_{k, t}} \sum_{i=1}^{N}|u|^{q_{i}} d x\right]
$$

for every $k \in \mathbf{N}$ and $R_{0} \leq \tau<t \leq R_{1}$, where $c_{0}$ is a positive constant that depends only on $N, q_{i}, r, R_{0}$, $R_{1}$ and $|\Omega|$ and $\gamma$ is a real positive constant. Then $u \in L_{\text {loc }}^{s}(\Omega)$, where

$$
s=\frac{\bar{q}^{*} q}{q-\bar{q}^{*}(1-1 / r)}
$$

One will also need a lemma from [8].

Lemma 2.2. Let $f(t)$ be a nonnegative bounded function defined for $0 \leq T_{0} \leq t \leq T_{1}$. Suppose that for $T_{0} \leq t<s \leq T_{1}$

$$
f(t) \leq A(s-t)^{-\gamma}+B+\theta f(s)
$$

where $A, B, \gamma, \theta$ are nonnegative constants, and $\theta<1$. Then there exists a constant $c$, depending only on $r$ and $\theta$ such that for every $\rho, R, T_{0} \leq \rho<R \leq T_{1}$, one has

$$
f(\rho) \leq c\left[A(R-\rho)^{-\gamma}+B\right]
$$




\section{Minima of anisotropic functionals}

In this section, we prove a local regularity result for minima of anisotropic functionals.

Definition 3.1. By a local minimum of the anisotropic functional $I$ in (1.3), we mean a function $u \in W_{\text {loc }}^{1, q_{i}}(\Omega)$, such that for every function $\psi \in W^{1, q_{i}}(\Omega)$ with supp $\psi \subset \subset \Omega$, it holds that

$$
I(u ; \operatorname{supp} \psi) \leq I(u+\psi ; \operatorname{supp} \psi) .
$$

Theorem 3.2. Assume that the functional I satisfies the conditions (1.7). If $u$ is a local minimum of I, then it belongs to $L_{\text {loc }}^{s}(\Omega)$, where

$$
s=\frac{\bar{q}^{*} q}{q-\bar{q}^{*}\left(1-1 / \min \left\{r_{1}, r_{2}\right\}\right)} .
$$

Proof. Owing to Lemma 2.1, it is sufficient to prove that $u$ satisfies the integral estimates (2.2) with $\gamma=q$ and $\phi_{0}=\varphi_{0}+\varphi_{1}$. Let $B_{R_{1}} \subset \subset \Omega$ and $0 \leq R_{0} \leq \tau<t \leq R_{1}$ be arbitrarily but fixed. It is no loss of generality to assume that $R_{1}-R_{0}<1$. For $k>0$, let

$$
A_{k}^{+}=\{x \in \Omega: u(x)>k\}, \quad A_{k}^{-}=\{x \in \Omega: u(x)<-k\} .
$$

It is obvious that $A_{k}=A_{k}^{+} \cup A_{k}^{-}$. Denote $A_{k, t}^{+}=A_{k}^{+} \cap B_{t}$ and $A_{k, t}^{-}=A_{k}^{-} \cap B_{t}$. Let $w=\max (u-k, 0)$. Choose $\psi=-\eta w$ in (3.1), where $\eta$ is a cut-off function such that

$$
\text { supp } \eta \subset B_{t}, \quad 0 \leq \eta \leq 1, \eta=1 \text { in } B_{\tau}, \quad|D \eta| \leq 2(t-\tau)^{-1} .
$$

We obtain from the minimality of $u$ that

$$
\begin{aligned}
\int_{B_{t}} f(x, u, D u) d x \leq \int_{B_{t}} f(x, u+\psi, D u+D \psi) d x \\
\quad=\int_{A_{k, t}^{+}} f(x, u-\eta w, D u-D(\eta w)) d x+\int_{B_{t} \cap\{u \leq k\}} f(x, u, D u) d x .
\end{aligned}
$$

This implies that

$$
\int_{A_{k, t}^{+}} f(x, u, D u) d x \leq \int_{A_{k, t}^{+}} f(x, u-\eta w, D u-D(\eta w)) d x
$$

By (1.7), we obtain

$$
\begin{aligned}
& \int_{A_{k, t}^{+}} \sum_{i=1}^{N}\left|\frac{\partial u}{\partial x_{i}}\right|^{q_{i}} d x \\
& \quad \leq b \int_{A_{k, t}^{+}} u^{\alpha} d x+\int_{A_{k, t}^{+}} \varphi_{0} d x+a \int_{A_{k, t}^{+}} \sum_{i=1}^{N}\left|\frac{\partial u}{\partial x_{i}}-\frac{\partial(\eta w)}{\partial x_{i}}\right|^{q_{i}} d x+b \int_{A_{k, t}^{+}}(u-\eta w)^{\alpha} d x+\int_{A_{k, t}^{+}} \varphi_{1} d x .
\end{aligned}
$$


We first estimate the 3rd term on the right-hand side of (3.7). Using the elementary inequality

$$
(a+b)^{q} \leq 2^{q-1}\left(a^{q}+b^{q}\right), \quad a, b \geq 0, q \geq 1
$$

we obtain

$$
\begin{aligned}
a \int_{A_{k, t}^{+}} \sum_{i=1}^{N}\left|\frac{\partial u}{\partial x_{i}}-\frac{\partial(\eta w)}{\partial x_{i}}\right|^{q_{i}} d x & =a \int_{A_{k, t}^{+} \backslash A_{k, \tau}^{+}} \sum_{i=1}^{N}\left|\frac{\partial u}{\partial x_{i}}-\frac{\partial(\eta w)}{\partial x_{i}}\right|^{q_{i}} d x \\
& \leq 2^{q-1} a \int_{A_{k, t}^{+} \backslash A_{k, \tau}^{+}} \sum_{i=1}^{N}\left[(1-\eta)^{q_{i}}\left|\frac{\partial u}{\partial x_{i}}\right|^{q_{i}}+\left|\frac{\partial \eta}{\partial x_{i}}\right|^{q_{i}} w^{q_{i}}\right] d x \\
& \leq 2^{q-1} a \int_{A_{k, t}^{+} \backslash A_{k, \tau}^{+}} \sum_{i=1}^{N}\left|\frac{\partial u}{\partial x_{i}}\right|^{q_{i}} d x+\frac{2^{2 q-1} a}{(t-\tau)^{q_{i}}} \int_{A_{k, t}^{+} \backslash A_{k, \tau}^{+}} \sum_{i=1}^{N} w^{q_{i}} d x \\
& \leq 2^{q-1} a \int_{A_{k, t}^{+} \backslash A_{k, \tau}^{+}} \sum_{i=1}^{N}\left|\frac{\partial u}{\partial x_{i}}\right|^{q_{i}} d x+\frac{2^{2 q-1} a}{(t-\tau)^{q}} \int_{A_{k, t}^{+} \backslash A_{k, \tau}^{+}} \sum_{i=1}^{N} u^{q_{i}} d x
\end{aligned}
$$

since $w^{q_{i}} \leq u^{q_{i}}$ in $A_{k, t}^{+}$and $t-\tau<1$. The summation of the 1 st and the 4 th terms on the righthand side of (3.7) can be estimated as

$$
b \int_{A_{k, t}^{+}} u^{\alpha} d x+b \int_{A_{k, t}^{+}}(u-\eta w)^{\alpha} d x \leq 2 b \int_{A_{k, t}^{+}} u^{\alpha} d x
$$

Substituting (3.9) and (3.10) into (3.7) yields

$$
\begin{aligned}
& \int_{A_{k, t}^{+}} \sum_{i=1}^{N}\left|\frac{\partial u}{\partial x_{i}}\right|^{q_{i}} d x \\
& \quad \leq \int_{A_{k, t}^{+}}\left(\varphi_{0}+\varphi_{1}\right) d x+2 b \int_{A_{k, t}^{+}} u^{\alpha} d x+2^{q-1} a \int_{A_{k, t}^{+} \backslash A_{k, \tau}^{+}} \sum_{i=1}^{N}\left|\frac{\partial u}{\partial x_{i}}\right|^{q_{i}} d x+\frac{2^{2 q-1} a}{(t-\tau)^{q}} \int_{A_{k, t}^{+} \backslash A_{k, \tau}^{+}} \sum_{i=1}^{N} u^{q_{i}} d x .
\end{aligned}
$$

We know from [8] that if $\tilde{u} \in W^{1, p}\left(B_{t}\right)$ and $|\operatorname{supp} \tilde{u}| \leq(1 / 2)\left|B_{t}\right|$, we then have the Sobolev inequality

$$
\left(\int_{B_{t}} \widetilde{u}^{p^{*}} d x\right)^{p / p^{*}} \leq c_{1}(N, p) \int_{B_{t}}|D \tilde{u}|^{p} d x
$$

Let

$$
\tilde{u}= \begin{cases}u, & x \in A_{k, t^{\prime}}^{+} \\ 0, & x \in \Omega \backslash A_{k, t}^{+}\end{cases}
$$


By assumption, $p \leq \alpha<p^{*}$, which implies

$$
\begin{aligned}
\int_{A_{k, t}^{+}} u^{\alpha} d x & =\int_{B_{t}} \tilde{u}^{\alpha} d x \leq\|\tilde{u}\|_{p^{*}}^{\alpha-p}\left|B_{t}\right|^{1-\alpha / p^{*}}\left(\int_{B_{t}} \tilde{u}^{p^{*}} d x\right)^{p / p^{*}} \\
& \leq c_{1}\|\tilde{u}\|_{p^{*}}^{\alpha-p}\left|B_{t}\right|^{1-\alpha / p^{*}} \int_{B_{t}}|D \tilde{u}|^{p} d x \\
& \leq c_{1}\|\tilde{u}\|_{p^{*}}^{\alpha-p}\left|B_{t}\right|^{1-\alpha / p^{*}} \max \left\{1,2^{p / 2-1}\right\} \int_{B_{t}} \sum_{i=1}^{N}\left|\frac{\partial \tilde{u}}{\partial x_{i}}\right|^{q_{i}} d x \\
& =c_{1}\|\tilde{u}\|_{p^{*}}^{\alpha-p}\left|B_{t}\right|^{1-\alpha / p^{*}} \max \left\{1,2^{p / 2-1}\right\} \int_{A_{k, t}^{+}} \sum_{i=1}^{N}\left|\frac{\partial u}{\partial x_{i}}\right|^{q_{i}} d x,
\end{aligned}
$$

provided that $|\operatorname{supp} \tilde{u}|_{B_{t}}|\leq(1 / 2)| B_{t} \mid$. We can choose $T$ so small that for $t \leq T$ we get

$$
c_{1}\|\tilde{u}\|_{p^{*}}^{\alpha-p}\left|B_{t}\right|^{1-\alpha / p^{*}} \max \left\{1,2^{p / 2-1}\right\} \leq \frac{1}{4 b} .
$$

It is obvious that

$$
k^{p^{*}}\left|A_{k}^{+}\right| \leq\|\tilde{u}\|_{p^{*}, \Omega^{\prime}}^{p^{*}}
$$

and therefore, there exists a constant $k_{0}$, such that for $k \geq k_{0}$, we have

$$
\left|A_{k}^{+}\right| \leq \frac{1}{2}\left|B_{T / 2}\right|
$$

For such values of $k$ we then have $|\operatorname{supp} \tilde{u}|<(1 / 2)\left|B_{T / 2}\right|$ and therefore, if $T / 2 \leq t \leq T$,

$$
\int_{A_{K, t}^{+}} u^{\alpha} d x \leq \frac{1}{4 b} \int_{A_{K, t}^{+}} \sum_{i=1}^{N}\left|\frac{\partial u}{\partial x_{i}}\right|^{q_{i}} d x .
$$

Thus, from (3.11) and, we get

$$
\begin{aligned}
& \int_{A_{k, t}^{+}} \sum_{i=1}^{N}\left|\frac{\partial u}{\partial x_{i}}\right|^{q_{i}} d x \\
& \quad \leq 2 \int_{A_{k, t}^{+}}\left(\varphi_{0}+\varphi_{1}\right) d x+2^{q} a \int_{A_{k, t}^{+} \backslash A_{k, \tau}^{+}} \sum_{i=1}^{N}\left|\frac{\partial u}{\partial x_{i}}\right|^{q_{i}} d x+\frac{2^{2 q} a}{(t-\tau)^{q}} \int_{A_{k, t}^{+} \backslash A_{k, \tau}^{+}} \sum_{i=1}^{N}|u|^{q i} d x .
\end{aligned}
$$

Suppose now $T / 2 \leq \rho \leq \tau<t \leq R \leq T$, we get

$$
\begin{aligned}
& \int_{A_{k, \rho}^{+}} \sum_{i=1}^{N}\left|\frac{\partial u}{\partial x_{i}}\right|^{q_{i}} d x \\
& \quad \leq 2 \int_{A_{k, R}^{+}}\left(\varphi_{0}+\varphi_{1}\right) d x+2^{q} a \int_{A_{k, \downarrow}^{+} \backslash A_{k, \rho}^{+}} \sum_{i=1}^{N}\left|\frac{\partial u}{\partial x_{i}}\right|^{q_{i}} d x+\frac{2^{2 q} a}{(t-\tau)^{q}} \int_{A_{k, R}^{+}} \sum_{i=1}^{N}|u|^{q_{i}} d x
\end{aligned}
$$


Adding to both sides $2^{q} a$ times the left-hand side, we get eventually

$$
\begin{aligned}
& \int_{A_{k, \rho}^{+}} \sum_{i=1}^{N}\left|\frac{\partial u}{\partial x_{i}}\right|^{q_{i}} d x \\
& \quad \leq \frac{2}{2^{q} a+1} \int_{A_{k, R}^{+}}\left(\varphi_{0}+\varphi_{1}\right) d x+\frac{2^{q} a}{2^{q} a+1} \int_{A_{k, t}^{+}} \sum_{i=1}^{N}\left|\frac{\partial u}{\partial x_{i}}\right|^{q_{i}} d x+\frac{2^{2 q} a}{2^{q} a+1} \cdot \frac{1}{(t-\tau)^{q}} \int_{A_{k, R}^{+}} \sum_{i=1}^{N}|u|^{q_{i}} d x
\end{aligned}
$$

we can now apply Lemma 2.2 to conclude that

$$
\int_{A_{k, \tau}^{+}} \sum_{i=1}^{N}\left|\frac{\partial u}{\partial x_{i}}\right|^{q_{i}} d x \leq c\left\{\frac{2}{2^{q} a+1} \int_{A_{k, t}^{+}}\left(\varphi_{0}+\varphi_{1}\right) d x+\frac{2^{2 q} a}{2^{q} a+1} \cdot \frac{1}{(t-\tau)^{q}} \int_{A_{k, t}^{+}} \sum_{i=1}^{N}|u|^{q_{i}} d x\right\}
$$

where $c$ depends only on $q$ and $a$.

Since $-u$ minimizes the functional

$$
\tilde{F}(v ; \Omega)=\int_{\Omega} \tilde{f}(x, v, D v) d x
$$

where $\tilde{f}(x, v, p)=f(x,-v,-p)$ satisfies the same growth conditions (1.7), inequality (3.22) holds with $u$ replaced by $-u$. We then conclude that

$$
\int_{A_{k, \tau}^{-}} \sum_{i=1}^{N}\left|\frac{\partial u}{\partial x_{i}}\right|^{q_{i}} d x \leq c\left\{\frac{2}{2^{q} a+1} \int_{A_{k, t}^{-}}\left(\varphi_{0}+\varphi_{1}\right) d x+\frac{2^{2 q} a}{2^{q} a+1} \cdot \frac{1}{(t-\tau)^{q}} \int_{A_{k, t}^{-}} \sum_{i=1}^{N}|u|^{q_{i}} d x\right\} .
$$

Adding (3.22) and (3.24) yields

$$
\int_{A_{k, \tau}} \sum_{i=1}^{N}\left|\frac{\partial u}{\partial x_{i}}\right|^{q_{i}} d x \leq c\left\{\frac{2}{2^{q} a+1} \int_{A_{k, t}}\left(\varphi_{0}+\varphi_{1}\right) d x+\frac{2^{2 q} a}{2^{q} a+1} \cdot \frac{1}{(t-\tau)^{q}} \int_{A_{k, t}} \sum_{i=1}^{N}|u|^{q_{i}} d x\right\} .
$$

This shows that $u$ satisfies estimates (2.2) with $\gamma=q$ and $\phi_{0}=\varphi_{0}+\varphi_{1}$. Theorem 3.2 follows from Lemma 2.1.

\section{Local solutions of anisotropic equations}

In this section, we prove a local regularity result for weak solutions of anisotropic equations. Let $u \in W_{\text {loc }}^{1, q_{i}}(\Omega)$ be a local solution of the anisotropic equation (1.4), where $\mathcal{A}: \Omega \times \mathbf{R} \times \mathbf{R}^{n} \rightarrow \mathbf{R}^{n}$ is a Caratheodory function satisfying the structural conditions (1.9) and (1.10).

Definition 4.1. By a weak solution of (1.4) we mean a function $u \in W_{\text {loc }}^{1, q_{i}}(\Omega)$, such that for every function $\psi \in W^{1, q_{i}}(\Omega)$ with supp $\psi \subset \subset \Omega$ it holds

$$
\int_{\operatorname{supp} \psi} \mathcal{A}(x, u, D u) \cdot D \psi d x=\int_{\operatorname{supp} \psi} f \cdot D \psi d x
$$

where $f=\left(f_{1}, f_{2}, \ldots, f_{N}\right)$. 
Theorem 4.2. Under the previous assumptions (1.9) and (1.10), if one assumes that $\varphi_{2} \in L_{\mathrm{loc}}^{r_{0}}(\Omega)$, $f_{i} \in L_{\mathrm{loc}}^{r_{i}}(\Omega), i=1,2, \ldots, N, k \in L_{\mathrm{loc}}^{r_{N+1}}(\Omega)$, and $r_{i}, i=0, \ldots, N+1$ satisfy

$$
1<r=\min _{1 \leq i \leq N}\left\{\frac{r_{i}}{q_{i}^{\prime}}, r_{0}, \frac{r_{N+1}}{p^{\prime}}\right\}<\frac{N}{\bar{q}}
$$

then $u \in L_{\text {loc }}^{s}(\Omega)$, where

$$
s=\frac{\bar{q}^{*} q}{q-\bar{q}^{*}(1-1 / r)}
$$

Proof. By virtue of Lemma 2.1, it is sufficient to prove that $u$ satisfies the integral estimates (2.2) with $\gamma=q$ and $\phi_{0}=\varphi_{2}+|k|^{p^{\prime}}+\sum_{i=1}^{N}\left|f_{i}\right|^{q_{i}^{\prime}}$. Let $B_{R_{1}} \subset \subset \Omega$ and $0 \leq R_{0} \leq \tau<t \leq R_{1}$ be arbitrarily but fixed. Assume again that $R_{1}-R_{0}<1$. Let $w=\max \{u-k, 0\}$. Choose $\psi=\eta w$ as a test function in (4.1), where the cut-off function $\eta$ satisfies the conditions (3.4). We obtain from Definition 4.1 that

$$
\int_{A_{k, t}^{+}} \mathcal{A}(x, u, D u) \cdot D(\eta w) d x=\int_{A_{k, t}^{+}} f \cdot D(\eta w) d x
$$

We now estimate the integrals in (4.4). Applying the assumption (1.9), we deduce from (4.4) that

$$
\begin{aligned}
b_{0} \int_{A_{k, \tau}^{+}} \sum_{i=1}^{N}\left|\frac{\partial u}{\partial x_{i}}\right|^{q_{i}} d x \leq & b_{1} \int_{A_{k, t}^{+}}|u|^{\alpha_{1}} d x+\int_{A_{k, t}^{+}} \varphi_{2} d x+\int_{A_{k, t}^{+}} f \cdot D u d x+\frac{2}{t-\tau} \int_{A_{k, t}^{+}}|f| w d x \\
& +\frac{2}{t-\tau} \int_{A_{k, t}^{+} \backslash A_{k, \tau}^{+}}|\mathcal{A}(x, u, D u)| w d x .
\end{aligned}
$$

The 3rd term on the right-hand side of the above inequality can be estimated as

$$
\int_{A_{k, t}^{+}} f \cdot D u d x=\int_{A_{k, t}^{+}} \sum_{i=1}^{N} f_{i} \cdot \frac{\partial u}{\partial x_{i}} d x \leq \varepsilon \int_{A_{k, t}^{+}} \sum_{i=1}^{N}\left|\frac{\partial u}{\partial x_{i}}\right|^{q_{i}} d x+\sum_{i=1}^{N} C\left(\varepsilon, q_{i}\right) \int_{A_{k, t}^{+}}\left|f_{i}\right|^{q_{i}^{\prime}} d x \text {. }
$$

By Young's inequality, the 4th term on the right-hand side of inequality (4.5) can be estimated as

$$
\frac{2}{t-\tau} \int_{A_{k, t}^{+}}|f| w d x \leq \frac{2^{q}}{(t-\tau)^{q}} \int_{A_{k, t}^{+}} \sum_{i=1}^{N}(u-k)^{q^{i}} d x+\sum_{i=1}^{N} \int_{A_{k, t}^{+}}\left|f_{i}\right|^{q_{i}^{\prime}} d x
$$

By (1.10), the last term on the right-hand side of (4.5) can be estimated as

$$
\frac{2}{t-\tau} \int_{A_{k, t}^{+} \backslash A_{k, \tau}^{+}}|\mathcal{A}(x, u, D u)| w d x \leq \frac{2}{t-\tau} \int_{A_{k, t}^{+} \backslash A_{k, \tau}^{+}}\left[b_{2} \sum_{i=1}^{N}\left|\frac{\partial u}{\partial x_{i}}\right|^{q_{i}-1}+b_{3}|u|^{\alpha_{2}}+k\right] w d x=I_{1}+I_{2}+I_{3} .
$$


Gao Hongya et al.

By Young's inequality, we derive that

$$
I_{1} \leq b_{2} \int_{A_{k, t}^{+} \backslash A_{k, \tau}^{+}} \sum_{i=1}^{N}\left|\frac{\partial u}{\partial x_{i}}\right|^{q_{i}} d x+\frac{b_{2} 2^{q}}{(t-\tau)^{q}} \int_{A_{k, t}^{+} \backslash A_{k, \tau}^{+}} \sum_{i=1}^{N}(u-k)^{q_{i}} d x
$$

Hölder's inequality and Young's inequality yield

$$
\begin{aligned}
I_{2} & \leq b_{3} \varepsilon \int_{A_{k, t}^{+} \backslash A_{k, \tau}^{+}}|u|^{\alpha_{2} p^{\prime}} d x+\frac{C(\varepsilon, p) 2^{p}}{(t-\tau)^{p}} \int_{A_{k, t}^{+} \backslash A_{k, \tau}^{+}}(u-k)^{p} d x \\
& \leq b_{3} \varepsilon \int_{A_{k, t}^{+} \backslash A_{k, \tau}^{+}}|u|^{\alpha_{2} p^{\prime}} d x+\frac{C(\varepsilon, p) 2^{p}}{N(t-\tau)^{q}} \int_{A_{k, t}^{+} \backslash A_{k, \tau}^{+}} \sum_{i=1}^{N}(u-k)^{q_{i}} d x,
\end{aligned}
$$

where $\varepsilon$ is a positive constant to be determined later. Further,

$$
I_{3} \leq \int_{A_{k, t}^{+} \backslash A_{k, \tau}^{+}}|k|^{p^{\prime}} d x+\frac{2^{q}}{N(t-\tau)^{q}} \int_{A_{k, t}^{+} \backslash A_{k, \tau}^{+}} \sum_{i=1}^{N}(u-k)^{q_{i}} d x
$$

Combining (4.6)-(4.11) with (4.5) yields

$$
\begin{aligned}
b_{0} \int_{A_{k, \tau}^{+}} & \sum_{i=1}^{N}\left|\frac{\partial u}{\partial x_{i}}\right|^{q_{i}} d x \\
\leq & \int_{A_{k, t}^{+}}\left(\varphi_{2}+|k|^{p^{\prime}}+\sum_{i=1}^{N}\left(C\left(\varepsilon, q_{i}\right)+1\right)\left|f_{i}\right|^{q_{i}^{\prime}}\right) d x+b_{1} \int_{A_{k, t}^{+}}|u|^{\alpha_{1}} d x+b_{3} \varepsilon \int_{A_{k, t}^{+}}|u|^{\alpha_{2} p^{\prime}} d x \\
& +\varepsilon \int_{A_{k, t}^{+}} \sum_{i=1}^{N}\left|\frac{\partial u}{\partial x_{i}}\right|^{q^{i}} d x+b_{2} \int_{A_{k, t}^{+} \backslash A_{k, \tau}^{+}} \sum_{i=1}^{N}\left|\frac{\partial u}{\partial x_{i}}\right|^{q^{i}} d x \\
& +\left(b_{2}+C(\varepsilon, p)+2\right) \frac{2^{q}}{(t-\tau)^{q}} \int_{A_{k, t}^{+}} \sum_{i=1}^{N}(u-k)^{q^{i}} d x
\end{aligned}
$$

Since $p \leq \alpha_{1}<p^{*}$, then as in the proof of Theorem 3.2, we know that there exist a sufficiently small $T$ and a sufficiently large $k_{0}$, such that for all $T / 2 \leq t \leq T$ and $k \geq k_{0}$, we have

$$
\int_{A_{k, t}^{+}}|u|^{\alpha_{1}} d x \leq \frac{1}{2 b_{1}} \int_{A_{k, t}^{+}} \sum_{i=1}^{N}\left|\frac{\partial u}{\partial x_{i}}\right|^{q_{i}} d x
$$

Similarly, since $p-1 \leq \alpha_{2} \leq N(p-1) /(n-p)$, then $p \leq \alpha_{2} p^{\prime} \leq p^{*}$, therefore

$$
\int_{A_{k, t}^{+}}|u|^{\alpha_{2} p^{\prime}} d x \leq C \int_{A_{k, t}^{+}} \sum_{i=1}^{N}\left|\frac{\partial u}{\partial x_{i}}\right|^{q_{i}} d x
$$


Thus, from (4.12)-(4.14) we can derive that

$$
\begin{aligned}
b_{0} \int_{A_{k, \tau}^{+}} \sum_{i=1}^{N}\left|\frac{\partial u}{\partial x_{i}}\right|^{q_{i}} d x \leq & \int_{A_{k, t}^{+}}\left(\varphi_{2}+|k|^{p^{\prime}}+\sum_{i=1}^{N}\left(C\left(\varepsilon, q_{i}\right)+1\right)\left|f_{i}\right|^{q_{i}^{\prime}}\right) d x \\
& +\left(\frac{1}{2}+\left(C b_{3}+1\right) \varepsilon\right) \int_{A_{k, t}^{+}} \sum_{i=1}^{N}\left|\frac{\partial u}{\partial x_{i}}\right|^{q i} d x+b_{2} \int_{A_{k, t}^{+} \backslash A_{k, \tau}^{+}} \sum_{i=1}^{N}\left|\frac{\partial u}{\partial x_{i}}\right|^{q_{i}} d x \\
& +\left(b_{2}+C(\varepsilon, p)+2\right) \frac{2^{q}}{(t-\tau)^{q}} \int_{A_{k, t}^{+}} \sum_{i=1}^{N}(u-k)^{q_{i}} d x .
\end{aligned}
$$

Adding to both sides

$$
b_{2} \int_{A_{k, \tau}^{+}} \sum_{i=1}^{N}\left|\frac{\partial u}{\partial x_{i}}\right|^{q_{i}} d x
$$

we get eventually

$$
\begin{aligned}
\int_{A_{k, \tau}^{+}} \sum_{i=1}^{N}\left|\frac{\partial u}{\partial x_{i}}\right|^{q_{i}} d x \leq & \frac{1}{b_{0}+b_{2}} \int_{A_{k, t}^{+}}\left(\varphi_{2}+|k|^{p^{\prime}}+\sum_{i=1}^{N}\left(C\left(\varepsilon, q_{i}\right)+1\right)\left|f_{i}\right|^{q_{i}^{\prime}}\right) d x \\
& +\left(\frac{1}{2}+\left(C b_{3}+1\right) \varepsilon\right) \frac{1}{b_{0}+b_{2}} \int_{A_{k, t}^{+}} \sum_{i=1}^{N}\left|\frac{\partial u}{\partial x_{i}}\right|^{q_{i}} d x+\frac{b_{2}}{b_{0}+b_{2}} \int_{A_{k, t}^{+}} \sum_{i=1}^{N}\left|\frac{\partial u}{\partial x_{i}}\right|^{q_{i}} d x \\
& +\left(b_{2}+C(\varepsilon, p)+2\right) \frac{1}{b_{0}+b_{2}} \frac{2^{q}}{(t-\tau)^{q}} \int_{A_{k, t}^{+}} \sum_{i=1}^{N}(u-k)^{q_{i}} d x .
\end{aligned}
$$

Choosing $\varepsilon$ small enough, such that

$$
\theta=\frac{1 / 2+\left(C b_{3}+1\right) \varepsilon+b_{2}}{b_{0}+b_{2}}<1
$$

(4.17) implies that

$$
\int_{A_{k, \tau}^{+}} \sum_{i=1}^{N}\left|\frac{\partial u}{\partial x_{i}}\right|^{q_{i}} d x \leq C \int_{A_{k, t}^{+}}\left(\varphi_{2}+|k|^{p^{\prime}}+\sum_{i=1}^{N}\left|f_{i}\right|^{q_{i}^{\prime}}\right) d x+\theta \int_{A_{k, t}^{+}} \sum_{i=1}^{N}\left|\frac{\partial u}{\partial x_{i}}\right|^{q_{i}} d x+\frac{C}{(t-\tau)^{q}} \int_{A_{k, t}^{+}} \sum_{i=1}^{N}(u-k)^{q_{i}} d x .
$$

Suppose now that $T / 2 \leq \rho \leq \tau<t \leq R \leq T$, we get

$$
\begin{aligned}
\int_{A_{k, \rho}^{+}} \sum_{i=1}^{N}\left|\frac{\partial u}{\partial x_{i}}\right|^{q_{i}} d x \leq & C \int_{A_{k, t}^{+}}\left(\varphi_{2}+|k|^{p^{\prime}}+\sum_{i=1}^{N}\left|f_{i}\right|^{q_{i}^{\prime}}\right) d x \\
& +\theta \int_{A_{k, R}^{+}} \sum_{i=1}^{N}\left|\frac{\partial u}{\partial x_{i}}\right|^{q_{i}} d x+\frac{C}{(R-\rho)^{q}} \int_{A_{k, t}^{+}} \sum_{i=1}^{N}(u-k)^{q_{i}} d x .
\end{aligned}
$$


Applying Lemma 2.2, we conclude that

$$
\int_{A_{k, \tau}^{+}} \sum_{i=1}^{N}\left|\frac{\partial u}{\partial x_{i}}\right|^{q_{i}} d x \leq c C \int_{A_{k, t}^{+}}\left(\varphi_{2}+|k|^{p^{\prime}}+\sum_{i=1}^{N}\left|f_{i}\right|^{q_{i^{\prime}}}\right) d x+\frac{c C}{(t-\tau)^{q}} \int_{A_{k, t}^{+}} \sum_{i=1}^{N}(u-k)^{q_{i}} d x .
$$

Since $-u$ is a weak solution of

$$
-\operatorname{div} \tilde{A}(x, u, D u)=-\sum_{i=1}^{N} \frac{\partial f_{i}}{\partial x_{i}}
$$

where $\tilde{A}(x, s, \xi)=\mathcal{A}(x,-s,-\xi)$ satisfies the same conditions (1.9) and (1.10), inequality (4.21) holds with $u$ replaced by $-u$. We then conclude that

$$
\int_{A_{k, \tau}^{-}} \sum_{i=1}^{N}\left|\frac{\partial u}{\partial x_{i}}\right|^{q_{i}} d x \leq c C \int_{A_{k, t}^{-}}\left(\varphi_{2}+|k|^{p^{\prime}}+\sum_{i=1}^{N}\left|f_{i}\right|^{q_{i}^{\prime}}\right) d x+\frac{c C}{(t-\tau)^{q}} \int_{A_{k, t}^{-}} \sum_{i=1}^{N}(u-k)^{q_{i}} d x .
$$

Adding (4.21) with (4.23) yields

$$
\int_{A_{k, \tau}} \sum_{i=1}^{N}\left|\frac{\partial u}{\partial x_{i}}\right|^{q_{i}} d x \leq c C \int_{A_{k, t}}\left(\varphi_{2}+|k|^{p^{\prime}}+\sum_{i=1}^{N}\left|f_{i}\right|^{q_{i}^{\prime}}\right) d x+\frac{c C}{(t-\tau)^{q}} \int_{A_{k, t}} \sum_{i=1}^{N}(u-k)^{q_{i}} d x .
$$

Thus, $u$ satisfies (2.2) with $\phi_{0}=\varphi_{2}+|k|^{p^{\prime}}+\sum_{i=1}^{N}\left|f_{i}\right|^{q_{i}^{\prime}}$ and $\alpha=q$. Theorem 4.2 follows from Lemma 2.1.

\section{Acknowledgments}

Gao Hongya is supported by Special Fund of Mathematics Research of Natural Science Foundation of Hebei Province (no. 07M003) and Doctoral Foundation of the Department of Education of Hebei Province (B2004103). Chu Yuming is supported by NSF of Zhejiang Province (No. Y607128) and NSFC (no. 10771195). This research was done when the first author was visiting Institute of Mathematics, Nankai University. He wished to thank the Institute for support and hospitality.

\section{References}

[1] O. A. Ladyženskaya and N. N. Ural'ceva, Linear and Quasilinear Elliptic Equations, Academic Press, New York, NY, USA, 1968.

[2] C. B. Morrey, Multiple Integrals in the Calculus of Variations, Springer, Berlin, Germany, 1968.

[3] D. Gilbarg and N. S. Trudinger, Elliptic Partial Differential Equations of Second Order, Springer, Berlin, Germany, 1977.

[4] M. Giaquinta, Multiple Integrals in the Calculus of Variations and Nonlinear Elliptic Systems, Annals of Mathematics Studies, no. 105, Princeton University Press, Princeton, NJ, USA, 1983.

[5] G. Stampacchia, Ėquations Elliptiques du Second Ordre á Coefficients Discontinus, Séminaire de Mathématiques Supérieures, Les Presses de l'Université de Montréal, Montreal, Quebec, Canada, 1966.

[6] L. Boccardo and D. Giachetti, "Alcune osservazioni sulla regolaritá delle soluzioni di problemi nonlineari e applicazioni," Ricerche di Matematica, vol. 34, pp. 309-323, 1985.

[7] D. Giachetti and M. M. Porzio, "Local regularity results for minima of functionals of the calculus of variation," Nonlinear Analysis. Theory, Methods \& Applications, vol. 39, no. 4, pp. 463-482, 2000.

[8] M. Giaquinta and E. Giusti, "On the regularity of the minima of variational integrals," Acta Mathematica, vol. 148, no. 1, pp. 31-46, 1982. 